El genitivo agente como la interpretación semántica más prototípica en inglés: un estudio de corpus

\title{
EL GENITIVO AGENTE COMO LA INTERPRETACIÓN SEMÁNTICA MÁS PROTOTÍPICA EN INGLÉS: UN ESTUDIO DE CORPUS ${ }^{1}$
}

\author{
Encarnación Almazán Ruiz \\ Universidad de Jaén (España) \\ ealmazan@ujaen.es
}

Recibido: 15/08/2020 - Aprobado: 01/10/2020 - Publicado: 15/04/2021

DOI: doi.org/10.17533/udea.lyl.n79a06

\begin{abstract}
Resumen: La construcción de genitivo en inglés [NP's + N] puede ser interpretada de diversas maneras atendiendo no solo a factores léxicos y semánticos, sino también pragmáticos. El objetivo principal de este estudio es plantear que el genitivo agente (John's claim) es más habitual en inglés que el de posesión (John's car), a pesar de lo que tradicionalmente se mantiene. El estudio demuestra este hecho analizando ejemplos de dos corpus de lengua escrita: el Brown y el Lobtag. Además de relacionar el genitivo agente con el proceso de nominalización, se ofrece una posible explicación de la frecuencia de esta interpretación semántica.
\end{abstract}

Palabras clave: genitivo inglés; nominalización; semántica; lingüística de corpus.

\section{THE AGENTIVE GENITIVE AS THE MOST PROTOTYPICAL SEMANTIC INTERPRETATION IN ENGLISH: A CORPUS-BASED STUDY}

\begin{abstract}
The English genitive construction [NP's + N] can be semantically interpreted in several ways, considering not only lexical and semantic factors but also pragmatic ones. The main aim of this paper is to state that the agentive genitive (John's claim) is more frequent than the possessive (John's car), despite what has been claimed traditionally. The corpusbased study demonstrates this fact analysing examples of genitive taken from two corpora: The Brown and the Lobtag. In addition to relating the agentive genitive to the nominalization process, a plausible explanation of the frequency of this semantic interpretation is provided.
\end{abstract}

Key words: English genitive; nominalization; semantics; corpus linguistics.

1. Nota aclaratoria: El presente artículo trata uno de los aspectos desarrollados en la tesis doctoral de la autora, cuyo título es Estudio morfosintáctico y semántico de la construcción de genitivo en inglés moderno basado en un estudio de corpus. Dirigida por el Prof. Dr. Juan Santana Lario, fue presentada y defendida en la Universidad de Granada, en 2005, para la obtención del Grado de Doctora en Filología Inglesa. 


\section{Introducción}

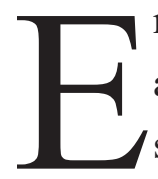

n el sistema de casos del inglés antiguo, el genitivo era el único que no designaba una función sintáctica argumental concreta, como era lo propio del resto de los casos gramaticales; el nominativo, por ejemplo,

se correspondía con el sujeto de la oración y el acusativo con el complemento directo; al genitivo, por el contrario, se le atribuían distintas explicaciones semánticas. Aunque en el inglés contemporáneo el genitivo ya no es un caso gramatical, bien es cierto que sigue relacionándose con numerosos significados o interpretaciones semánticas, de tal manera que Peter's train puede interpretarse como: el tren que Peter coge habitualmente, el tren que Peter ha diseñado o el tren - un juguete- que Peter posee. El que se entienda de un modo u otro depende de diferentes factores (Huddleston \& Pullum, 2002; Vikner \& Jensen, 2002) que están directamente vinculados a la relación que se establece entre el sintagma en genitivo y el núcleo del sintagma superordinado por medio de la marca ['s].

Tradicionalmente, el genitivo ha sido asociado al significado de posesión; de hecho, en ocasiones, se utilizan indistintamente los términos caso genitivo y caso posesivo para referirse a esta construcción, lo que da la idea de la íntima conexión de la misma al significado de posesión. No obstante, la posesión no es el único significado que se atribuye al genitivo. No solo las gramáticas más antiguas y tradicionales (Curme 1935, 1947; Nesfield, 1912; Quirk et al. 1985), sino también las de corte estructuralista (Christophersen \& Sandved, 1969; Sledd, 1959) reconocen otras interpretaciones semánticas como el genitivo de sujeto o genitivo agente, genitivo de objeto o afectado y genitivo descriptivo, entre otras. En realidad, el genitivo presentaba esta peculiaridad ya incluso en el indoeuropeo y en los períodos más antiguos del inglés, porque era el único caso que no estaba asociado a una función sintáctica, como el resto de casos, sino que siempre ha estado relacionado con el hecho de establecer una relación entre dos entidades que puede conllevar diferentes interpretaciones semánticas (Hogg, 1992) De entre todas las lecturas semánticas que se pueden hacer de la construcción de genitivo, el objeto de estudio en estas páginas es la interpretación semántica de genitivo agente.

El objetivo principal de este estudio es, pues, plantear que la interpretación semántica de genitivo agente resulta más frecuente y prototípica de lo que las distintas gramáticas hacen suponer. Asimismo, se tratará de establecer una relación entre el genitivo agente, en particular, y la construcción de genitivo, en general, con el fenómeno lingüístico denominado nominalización, observando que ambos pueden estar relacionados con el mecanismo del empaquetado de la información. 
El genitivo agente como la interpretación semántica más prototípica en inglés: un estudio de corpus

\section{El genitivo agente ${ }^{2}$}

Cuando se utiliza el término de genitivo agente se alude al hecho de que la relación semántica que se establece entre el sintagma en genitivo y el núcleo superordinado es similar a la que puede existir entre el sujeto y el verbo de una oración activa, de modo que el sujeto suele identificar a la persona que realiza la acción indicada por el verbo. Los ejemplos en (1) pueden interpretarse como genitivos agentes, mientras que, en las frases análogas de estos mismos, que se presentan en (1'), se puede observar esta relación.

(1) Her parents' consent

John's claim

Herbie's love

(1') Her parents consented something John claimed something

Herbie loves someone

Se debe señalar el hecho de que los núcleos superordinados de los ejemplos en (1) son nombres que proceden de un verbo (consent < to consent, claim < to claim, love < to love), lo que se conoce con el término deverbal nouns. Un «nombre procedente de un verbo» hace referencia a una relación y a una entidad o entidades que participan en dicha relación (Taylor, 1994). Esta construcción en la que aparece el genitivo junto a un nombre relacional ha sido objeto de estudio durante años y se engloba dentro de un fenómeno lingüístico conocido como nominalización (nominalization).

Según Crystal (1997), el término nominalización puede referirse tanto al proceso de formar un nombre desde otra clase de palabra (redness, refusal), como a la derivación de un sintagma nominal a partir de una oración subyacente (Her answering of the letter... from She answered the letter); por lo tanto, es de especial relevancia para este estudio la observación del segundo proceso.

Dentro de la literatura lingüística, este fenómeno ha sido estudiado por las distintas escuelas, encontrando aportaciones dentro de la gramática tradicional (Quirk et al., 1985), generativa (Chomsky, 1970), funcional (Eggins, 1994) y cognitiva (Langacker, 1991). De entre todas estas aportaciones, uno de los textos con quizás más trascendencia sobre este tema es Remarks on Nominalization, de Noam Chomsky (1970), representando igualmente uno de los estudios más importantes dentro de la corriente generativo-transformacional. En este estudio, Chomsky (1970, p. 187) propone que los siguientes ejemplos no se derivan de una transformación, como había sido la creencia hasta entonces, sino que necesariamente tienen que generarse en la base.

\footnotetext{
2. A pesar de lo controvertido y discutible que puede resultar el término genitivo, se ha optado por esta terminología por resultar más general y menos comprometida que la expresión sintagma posesivo, aclarando, no obstante, que en el inglés actual el término genitivo ya no es sinónimo de caso. En este artículo, el término posesivo hace referencia a una de las posibles interpretaciones semánticas que esta estructura puede tener y, por ello, resultaría más restrictivo. Asimismo, el término agente se considera el más apropiado para referirse a lo que las gramáticas tradicionales denominan como «subjective genitive» (Quirk et al., 1985, p. 321), porque aquel hace referencia al rol semántico, mientras que el de «sujeto» estaría relacionado con la función sintáctica.
} 
(2) John's refusal of the offer

John's criticism of the book

Por ello, Chomsky propuso las siguientes «reglas de estructura» (phrase structure rules):

\section{$\mathrm{NP} \rightarrow \mathrm{DET} \quad \mathrm{N}^{\prime}$ \\ $\mathrm{DET} \rightarrow \mathrm{NP} \quad$ POSS}

Como se puede observar, estas reglas determinan que el sintagma en genitivo (NP's) tiene estatus de determinante. Según Taylor (1996), las reglas habrían sido consistentes solo si Chomsky hubiera generado todos los tipos de genitivo de ellas. Sin embargo, aparte de los genitivos que se derivan de estas reglas, el mismo autor reconoció dos tipos más de genitivos prenominales que se generan por medio de transformaciones:

(A) Genitivos que denotan posesión alienable. Este tipo de genitivo se deriva de una oración de relativo en la que aparece el verbo to have.

(3) John's car $<$ The car that John has

(B) Genitivos que denotan posesión inalienable. Según Chomsky (1970), este tipo de expresiones sí se generan en la base por medio de las reglas descritas anteriormente y conllevan una conexión intrínseca.

(4) John's leg $\rightarrow$ The leg that is part of John's body.

(C) Genitivos que se derivan por medio del cambio de orden del sintagma nominal (NP-preposing). La derivación de este tipo de expresiones se puede explicar por analogía con la transformación de una oración pasiva que procede de una activa y por medio del movimiento del complemento directo a la posición de sujeto. Este tipo de ejemplos tendría una interpretación de genitivo de objeto (objective reading).

(5) The city's destruction by the enemy < the destruction of the city by the enemy

Por el contrario, como se ha señalado anteriormente, cuando se hace una lectura de genitivo agente (subjective reading), los ejemplos entonces son generados en la base por medio de las reglas anteriores.

(6) The enemy's destruction of the city $\rightarrow$ the enemy destroyed the city

Las nominalizaciones que estudia Chomsky están constituidas por nombres que implican algún tipo de relación y que, al menos semánticamente, se puede decir que proceden de un verbo (deverbal noun), aunque no necesariamente desde un punto de vista formal o morfológico. Asimismo, Langacker (1991) distinguió distintos tipos de esta clase relacional de nombres:

3. $\quad$ Barker (1995, p. 67) señaló que el contraste entre «alienable e inalienable es una distinción gramatical que existe en algunas lenguas y que es marcada por medio de varios procedimientos de carácter morfológico o sintáctico». Sin embargo, la lengua inglesa muestra muy poca inclinación a hacer una distinción gramatical entre la posesión alienable y la inalienable. Según este autor, los nombres típicamente considerados inalienables, como son las partes del cuerpo y los términos de parentesco, denotan relaciones, mientras que los reconocidos como alienables indican característicamente una clase. 
El genitivo agente como la interpretación semántica más prototípica en inglés: un estudio de corpus

Nombres agentes (agent nouns). Aparecen normalmente marcados por el sufijo -er y designan no solo a quien hace una acción, sino la acción en sí misma.

(7) Singer, invader, narrator

Singer $\rightarrow$ the person who sings

Nombres pacientes (patient nouns). El sufijo que suele caracterizarlos es -ee y designa a la persona que recibe la acción.

\section{(8) Draftee, appointee}

Nombres de resultado (result nouns). Designan una entidad que es la consecuencia de un proceso. No suelen tener marca morfológica que los distinga.

(9) Photograph, bruise

Nombres de modo (manner nouns). Sin una marca morfológica que los caracterice, estos nombres suelen designar el modo en el que un proceso o acción se lleva a cabo por el agente. Langacker pone como ejemplo el nombre walk en las siguientes oraciones:

(10) His walk is peculiar.

He had a peculiar walk.

Nombres de habilidad (ability nouns). Designan la habilidad del agente para realizar una actividad concreta, como por ejemplo speech.

(11) His speech returned.

He lost his speech.

Nombres episódicos (episodic nouns). Hacen real o concreto un episodio del proceso. Al mismo tiempo, tanto el agente (trajector) como el elemento destacado (landmark) son degradados a elementos de la predicación.

La clasificación anterior no debe entenderse como distintiva ni definitiva, pues muchos nombres de este tipo (deverbal nouns) son polisémicos con respecto a más de una de las categorías señaladas y resulta difícil establecer una clara distinción conceptual; por ejemplo, entre un nombre episódico y un nombre de resultado o paciente.

Si se toma por caso el nombre destruction, se nota que puede tener varias interpretaciones:

(12) The city's destruction continued for three days. $\rightarrow$ Actividad

The destruction that the enemy caused will take many months to repair. $\rightarrow$ Resultado

Cuando destruction se interpreta como actividad, the city's destruction solo puede tener una interpretación de objeto (objective reading), pues obviamente la ciudad es destruida por alguien o por algo. Sin embargo, cuando destruction se analiza como resultado, el sintagma en genitivo también admite una interpretación como agente. Esto se debe a que cuando se considera un nombre de resultado, destruction denota al elemento más destacado el cual resulta del proceso y no indica un episodio del proceso en sí. Si se ve la lectura del agente en los siguientes 
El genitivo agente como la interpretación semántica más prototípica en inglés: un estudio de corpus ejemplos, se nota claramente que los sintagmas en genitivo (the bomb; the enemy) denotan al causante de la destrucción:

(13) The bomb's destruction extended over several blocks (Agente).

The enemy's destruction took many months to repair (Agente).

El interpretar que ambos sintagmas en genitivo designan al causante de la destrucción puede resultar, $a$ priori, una interpretación demasiado general y arriesgada desde el punto de vista lingüístico. Indudablemente, la propiedad de [+ animado] en el nombre enemy hace más evidente esta interpretación que en el caso de bomb, ya que este nombre designa una entidad claramente no volitiva y que, por tanto, tendría que interpretarse como instrumental $^{4}$ en lugar de agente. Sin embargo, se ha tomado esta determinación porque en estas páginas no se están dilucidando los diferentes roles semánticos que se le pueden atribuir al sujeto de una oración, sino que se está proponiendo una relación entre el sintagma en genitivo y el núcleo superordinado análoga a la que se puede establecer en una oración activa entre el sujeto agente y la acción del verbo.

Con respecto a las nominalizaciones, McCawley (1998) señaló que, aunque en ocasiones tienen formas que recuerdan a las «nominalizaciones en -ing», las primeras difieren de estas en la estructura superficial, ya que el verbo nominalizado es el núcleo de un grupo nominal cuya estructura interna es la típica de un sintagma nominal (NP), mientras que la de una «nominalización en -ing» recuerda más a la estructura interna de una oración. Si se revisan los siguientes ejemplos que el propio autor propuso:

(14) a. John's consumption of liquor

b. John's consuming liquor

McCawley (1998) los analizó del siguiente modo:

4. Alexiadou and Schäfer (2006, p. 42) explicaron que a pesar de que los sujetos de carácter instrumental son plausibles en la lengua inglesa, determinar por qué solo algunos instrumentos pueden aparecer como sujetos en una oración conlleva hacer una distinción entre dos tipos de instrumentos: los puros por naturaleza (pure instruments) y los que se pueden clasificar como causantes (instruments causers). El considerar esta distinción en este estudio habría supuesto el establecer más paradigmas dentro del análisis del corpus, algo que habría excedido, sin duda, los límites de estas páginas. 
El genitivo agente como la interpretación semántica más prototípica en inglés: un estudio de corpus

a.

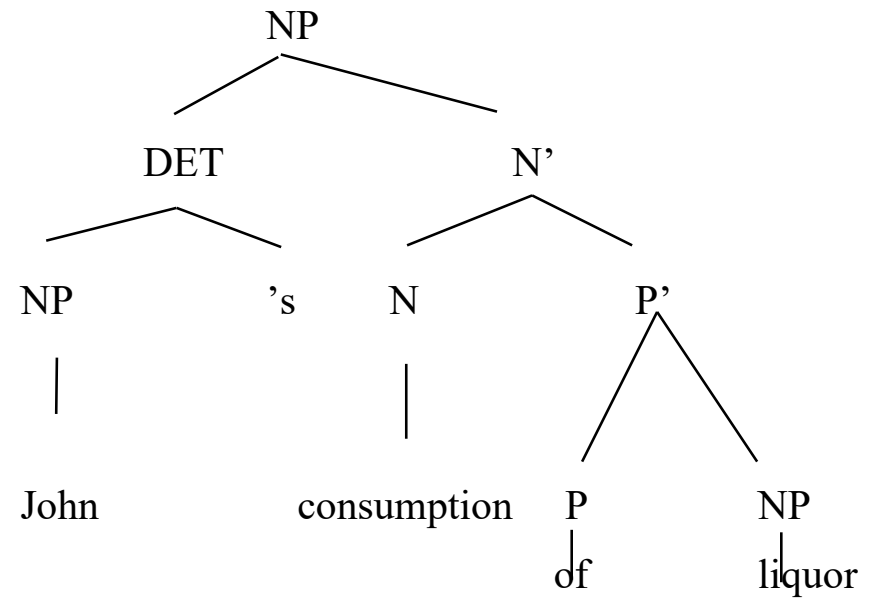

b.

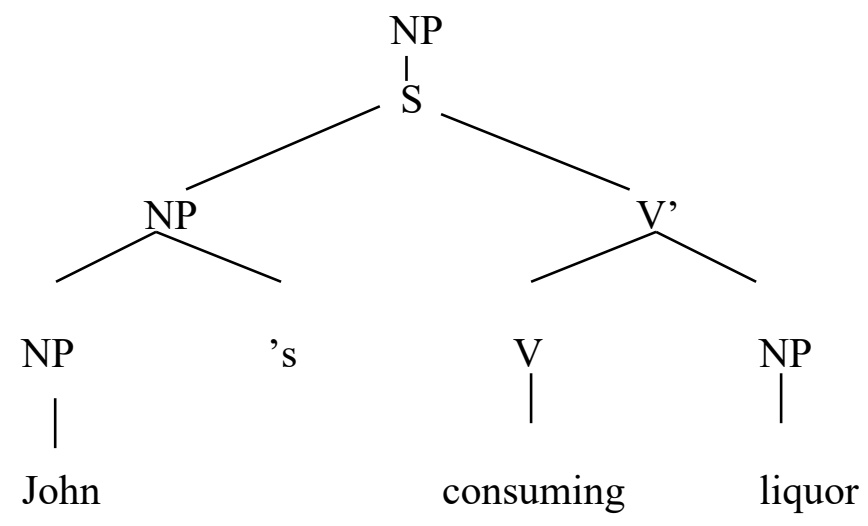

En ese sentido, McCawley (1998) indicó igualmente algunas diferencias entre los dos tipos de nominalizaciones:

(A) En las nominalizaciones con nombres derivados de un verbo como núcleos superordinados, cualquier sintagma nominal dependiente debe ser introducido por una preposición.

(15) a. John's refusal of our request

b. John's refusing (*of) our request left us in a serious difficulty.

(B) Los núcleos de las nominalizaciones pueden aparecer en plural, menos las «nominalizaciones en -ing».

(16) a. Brendel's performances of the Beethoven sonatas

b. * Brendel's performings Boulez's works astonished the critics.

(C) Los núcleos de las nominalizaciones pueden estar modificados por adjetivos o por oraciones restrictivas.

(17) a. Beethoven's greatest compositions

b. Beethoven's (*admirable) composing his ninth symphony created a great stir. 
(D) El núcleo de una nominalización no puede ser la forma nominalizada de un verbo auxiliar.

(18) a. * Smith's be awarded of a medal.

b. The ceremony ended with Smith's being awarded a medal.

Aunque en este apartado se han tratado las nominalizaciones en relación con el genitivo, así como con la presencia de núcleos realizados por nombres que semánticamente se derivan de un verbo, hay que dejar constancia que para entender un sintagma en genitivo como agente no es totalmente imprescindible que el núcleo superordinado sea una nominalización o un nombre derivado de un verbo, sino que en muchos casos es suficiente con que dicho núcleo sea un nombre de acción, es decir, un nombre capaz de aludir a acciones o a resultados de dichas acciones para los que, eventualmente, también puede existir un verbo.

(19) John's victory $\Rightarrow$ John won.

En este ejemplo, victory difícilmente puede considerarse como una nominalización del verbo to win, así como tampoco puede entenderse como una forma nominal procedente de dicho verbo; sin embargo, es innegable que están relacionados semánticamente, ya que aluden al mismo tipo de acción o resultado.

En definitiva, como se ha puesto de manifiesto, en las nominalizaciones donde aparecen nombres semánticamente derivados de verbos en función de núcleos del sintagma, se establece una relación entre el sintagma en genitivo y el núcleo superordinado, entendiéndose, generalmente, que el genitivo realiza la acción implícita en el nombre. Asimismo, se ha expuesto que para entender el genitivo como agente no es necesario que aparezca una nominalización propiamente dicha, sino que en ocasiones basta con que haya implícita una acción.

\section{Metodología}

Los corpus informatizados se han venido utilizando durante las últimas décadas, resultando útiles no solo para la investigación lingüística, sino también para la filosófica, estilística y psicológica, entre otras. Por esta razón, para la selección del material que sirvió de base empírica para esta investigación, se utilizaron dos corpus informatizados: el Brown corpus y el $L O B$ corpus, ambos de acceso público. De estos dos corpus, el primero en recopilarse fue el Brown Corpus, conocido oficialmente como The Brown University Standard Corpus of PresentDay American English (Kučera \& Francis, 1967). Este corpus consiste en la recopilación de textos en inglés americano publicados durante 1961, realizado en la Universidad de Brown (Providence, Rhode Island, EE UU), siendo su principal objetivo contribuir a la investigación lingüística. El otro corpus que se empleó fue el conocido como el LOB Corpus, aunque su nombre completo es The Lancaster/Oslo-Bergen Corpus of British English (Johansson et al., 1978). Se trabajó entonces con la versión etiquetada, en la que cada palabra va acompañada de una etiqueta (tag) que indica la categoría gramatical o clase de palabra a la que pertenece cada palabra; por esta razón, a esta versión del corpus se le dio el nombre de Lobtag Corpus. Como dato importante, se menciona que 
El genitivo agente como la interpretación semántica más prototípica en inglés: un estudio de corpus la extensión de ambos corpus es de aproximadamente un millón de palabras.

Aunque hay corpus, tanto de inglés hablado como escrito, más recientes y con mayor número de palabras, la razón principal para basar este estudio en el Brown Corpus y en el Lobtag Corpus es poder contar con un corpus de ejemplos de la construcción de genitivo lo suficientemente representativo como para lograr que los resultados fuesen lo más concluyentes posibles. El hecho que ambos corpus puedan ser considerados pequeños por su número de palabras, en comparación con otros corpus más recientes, no fue motivo de peso para descartarlos, porque el valor de un corpus, como afirmó Anthony (2013) no se halla en el tamaño, sino en qué tipo de información se puede obtener del mismo. Además, el hecho de que ambos corpus contengan un millón de palabras ofreció dos ventajas: por un lado, proporcionaron un corpus representativo en cuanto a número de ejemplos y por otro, facilitó el proceso de análisis del mismo, pues se ha podido obtener un corpus manejable en cuanto a número de datos. Asimismo, teniendo en cuenta que la lengua inglesa no ha sufrido modificaciones importantes en el último medio siglo en cuanto a estructuras morfosintácticas se refiere, el hecho de que ambos corpus no sean los más recientes no supuso un argumento para desestimarlos, pues es innegable que ambos son todavía una clara muestra del inglés contemporáneo. Así, en la recopilación del corpus se tuvo en cuenta la estructura [NP's N] y para ello se hizo uso del programa informático Wordcruncher ${ }^{5}$, el cual permite hacer búsquedas y confrontaciones de manera rápida, por lo que resultó útil y ventajoso para la investigación.

Con el propósito mencionado anteriormente - de obtener un corpus representativo en cuanto a número de ejemplos y también con el de no alterar el material a estudiar-, se consideraron todos los ejemplos de la estructura de genitivo obtenidos tras la búsqueda automática de la secuencia *'s, donde el asterisco significa que el programa debía suministrar una lista de cualquier palabra que terminara en ['s]. Tras la búsqueda automática, se suprimieron todos los ejemplos que no fuesen sintagmas en genitivo del Brown Corpus. Igualmente, del Lobtag Corpus se descartaron todos los resultados que, a pesar de estar etiquetados como posesivos, no eran relevantes para el presente estudio por tratarse de pronombres y determinantes posesivos. De este modo, del Brown Corpus se contó con 4960 muestras de la construcción de genitivo, mientras que del Lobtag Corpus se obtuvo 4 765, los cuales con un total de 9725 ejemplos conformaron el corpus completo para el análisis.

\section{Análisis y discusión de los resultados}

\subsection{El genitivo agente en el corpus}

En este apartado se presentarán los datos obtenidos en el estudio de corpus que se realizó con respecto al genitivo agente a la vez que se analizan los resultados. El análisis del corpus revela que esta interpretación semántica del genitivo es la más frecuente en ambos corpus, ya que de los ejemplos de genitivo analizados en el Brown corpus, el 42,6 \% corresponde al genitivo agente, mientras que en el Lobtag corpus resultó ser el 41,2\%

5. http://wordcruncher.com/ 
El genitivo agente como la interpretación semántica más prototípica en inglés: un estudio de corpus de ellos.

Algunos autores como Hawkins (1981) o Lyons (1986) denominaron la construcción de genitivo como sintagma posesivo (possessive phrase), haciendo así especial referencia a una de las interpretaciones semánticas que el genitivo puede tener. Sin embargo, esta nomenclatura no resultaría del todo apropiada, pues excluiría el resto de interpretaciones semánticas que, como es bien sabido, el genitivo puede tener. A este respecto, el análisis del corpus indica que la lectura semántica de posesión no es la más frecuente en los ejemplos estudiados. La Tabla 1 muestra los datos comparativos del genitivo agente y del genitivo de posesión.

\begin{tabular}{|c|c|c|c|c|}
\hline \multicolumn{1}{c}{} & \multicolumn{2}{c}{ Brown Corpus } & \multicolumn{2}{c|}{ Lobtag Corpus } \\
\cline { 2 - 5 } & \multicolumn{1}{c}{$\begin{array}{c}\text { Frecuencia } \\
\text { absoluta }\end{array}$} & $\begin{array}{c}\text { Frecuencia } \\
\text { relativa }\end{array}$ & $\begin{array}{c}\text { Frecuencia } \\
\text { absoluta }\end{array}$ & $\begin{array}{c}\text { Frecuencia } \\
\text { relativa }\end{array}$ \\
\cline { 2 - 6 } & 2117 & $42,6 \%$ & 1967 & $41,2 \%$ \\
\hline Genitivo agente & 1373 & $27,7 \%$ & 1286 & $27 \%$ \\
\hline
\end{tabular}

Tabla 1. Frecuencias del genitivo agente y de posesión

Como demuestran los datos anteriores, no hay diferencias significativas entre las dos variedades del inglés analizadas. Asimismo, los datos determinan que en ambas la lectura semántica de genitivo agente es más habitual que la de posesión 6 .

No obstante, hay que aclarar que hemos interpretado como genitivos agentes no solo aquellos ejemplos en los que aparece un nombre derivado de un verbo como núcleo superordinado, sino también aquellos otros en los que se sobreentiende una acción, aunque esta no aparezca de forma explícita. Estos últimos ejemplos se corresponden con lo que Quirk et al. (1985) denominaron como «genitivo de origen» (genitive of origin) y en este estudio se han incluido dentro del genitivo agente, porque, en realidad, se interpreta que el sintagma en genitivo realiza una acción, aunque esta no esté formal y explícitamente en el ejemplo.

En los siguientes ejemplos, extraídos del corpus elaborado en esta investigación, en unos aparecen nombres de acción y nombres derivados de verbos (20) como núcleos superordinados, mientras que en otros (21) esto no es así, lo que comprueba cómo en ambos casos se pueden interpretar como genitivos agentes.

(20) Caldwell's resignation $\rightarrow$ Caldwell resigned.

Formby's plan $\rightarrow$ Formby planned something.

The secretary's greatest achievement $\rightarrow$ The secretary achieved something.

Berger's direction $\rightarrow$ Berger directed something.

Tring's campaign over property $\rightarrow$ Tring campaigned over property.

6. $\quad$ El resto de interpretaciones semánticas que se han tenido en cuenta en el análisis del corpus y que completarían la frecuencia relativa en cada uno de los corpus son las siguientes: Brown: afectado: 5,5 \%; relación personal: 4,6 \%; atributivo: 3,7 \%; descriptivo: 4,9\%; lugar: 6,7 \%; tiempo: 3,8 \%; idiomático: 0,5\% Lobtag: afectado: 5,5\%; relación personal: $6 \%$; atributivo: $3,8 \%$; descriptivo: 6,7 \%; lugar: 4,4\%; tiempo: 4,8\%; idiomático: 0,6\%. 
El genitivo agente como la interpretación semántica más prototípica en inglés: un estudio de corpus

(21) Keats' words $\rightarrow$ Keats said these words.

Slocum's telegram to Stanton $\rightarrow$ Slocum sent a telegram to Stanton.

Hardy's best poems $\rightarrow$ The best poems written by Hardy.

The novelist's carping phrase $\rightarrow$ The novelist said/wrote this phrase.

Mr. Wesker's message $\rightarrow$ Mr. Wesker sent/wrote a message.

Asimismo, en los ejemplos de genitivo que han sido interpretados como genitivos agentes cabe hacer la distinción, por un lado, de aquellos casos en los que aparece un nombre de acción o un nombre derivado de un verbo como núcleo superordinado y, por otro, distinguir aquellos otros en los que no. Atendiendo a esta apreciación los resultados han sido los siguientes:

(a) Por lo que al Brown corpus se refiere, cabe señalar que en el 55,3 \% de las muestras en genitivo clasificadas como agentes, el núcleo superordinado está realizado por un nombre de acción o por un nombre derivado de un verbo, como en (20), frente al 44,7 \% de los ejemplos. en los que esta clase de nombres no aparece en la función de núcleo, sino con otros sustantivos, como en (21).

(b) En el Lobtag corpus, la diferencia entre ambos porcentajes es un poco mayor, aunque de nuevo resulta algo mayor el porcentaje de ejemplos $(56,4 \%)$ que contienen un nombre procedente de un verbo como núcleo superordinado frente al 43,6 \% de los ejemplos en los que aparecen como núcleos otra clase de nombres.

Hasta ahora, se han clasificado los ejemplos de genitivo agente teniendo en cuenta si el núcleo superordinado del sintagma se podía derivar de algún verbo, si denotaba algún tipo de acción o si simplemente la acción se sobreentendía. Atendiendo a los ejemplos clasificados como agentes, se pasará a ver qué datos ofrece el corpus respecto a los rasgos semánticos del núcleo subordinado.

De los 2117 ejemplos clasificados como genitivo agente en el Brown corpus, el 82,3\% de ellos tienen como núcleo subordinado un nombre con el rasgo [+ humano]. Asimismo, en el Lobtag corpus se hallaron 1 967 resultados catalogados con este rasgo semántico, de los cuales el 85,9 \% presentaron un nombre animado y humano como núcleo del sintagma subordinado. Si se ven algunas muestras de ambos corpus:

(22) Brown corpus

Mr. Barcus' strong beliefs

Mossberg's latest contribution to the field

Volta's discovery of chemical electricity

His wife's desires

The doctor's return

The slave's dying words about the woman

(23) Lobtag corpus

Mr. Godber's speech

President Kennedy's suggestion

Robin Hood's famous advice

The singer's words

The maker's recommendation

The teacher's approach

Del elevado porcentaje de ejemplos con un nombre [+ humano] como núcleo subordinado, se puede hacer una subclasificación atendiendo a qué clase de nombre es dicho núcleo, es decir, si es un nombre propio o un 
El genitivo agente como la interpretación semántica más prototípica en inglés: un estudio de corpus

nombre común. El resultado en el Brown corpus es el siguiente: de los 1740 resultados que presentan un núcleo [+ humano], en el 74,3\% el núcleo es un nombre propio de persona, frente al $25,7 \%$, en los que el núcleo es un nombre común. Por su parte, el Lobtag corpus revela resultados muy similares en cuanto a esta subclasificación se refiere, puesto que de las 1697 muestras de genitivo agente con un nombre [+ humano] como núcleo subordinado, en el 70,8 \% el núcleo es un nombre propio de persona, mientras que solo en el 29,2 \% es un nombre común. A continuación, se ofrecerán algunos de estos ejemplos:

(A) Nombre propio [+ humano] como núcleo subordinado:

(24) Dr. Jenkins's conversation Jorda's return Mercer's explanation Alex's inspection Mr. Khrushchev's choice of alternatives Robby's performance last year

(B) Nombre común [+ humano] como núcleo subordinado:

(25) The Soviet leader's real aims His mother's disapproval The conductor's insistence The wife's attempt at control A boy's love for his mother The hearing officer's report

En ese sentido, también hay algunos ejemplos en los que el núcleo subordinado es un nombre [- animado] y [humano], que representan el 17,5\% de los ejemplos de genitivo agente en el Brown corpus y 13,1\% en el Lobtag corpus, como puede observarse en (26) y (27):

(26) Brown corpus

The company's contributions

The region's relative lack of purchasing power

London's approach in these discs

The country's development

The Earth's rotation

Russia's detonation of a super bomb

(27) Lobtag corpus

Britain's moves

The nation's greatest need

The world's reactions

Moscow's concern

The government's proposals

The heart's movements

De nuevo, dentro de este grupo de ejemplos con núcleo subordinado [- animado] y, por tanto, no humano, se diferenciaron otros subconjuntos, atendiendo a si se trata de un nombre propio o común. Se ofrecen algunos casos: 
El genitivo agente como la interpretación semántica más prototípica en inglés: un estudio de corpus

(C) Nombre propio inanimado como núcleo subordinado:

(28) Russia's progress

The United States' global commitments

West Germany's astonishing industrial rebirth

Britain's imports of food

America's determination to use her nuclear weapons

Somalia's political progress

(D) Nombre común inanimado como núcleo subordinado:

(29) The council's annual meeting

The board's action

The party's claim

The government's proposals

The blood's passage round the body

Your heart's desire

Como se puede apreciar en los ejemplos presentados en (29), algunos núcleos como council, board, party, y government pueden relacionarse con la actividad humana; por ello, se determinó otra subclasificación para comprobar si estos son los nombres inanimados más frecuentes o si, por el contrario, son más habituales los nombres como dream o heart.

El análisis del corpus ofrece los siguientes resultados a este respecto: en el Brown corpus, de los 371 ejemplos de genitivo agente con un nombre inanimado como núcleo subordinado, en el 63,7 \% de los casos, dichos núcleos se pueden vincular con la actividad humana, mientras que en el 36,3 \% restante esta relación no tendría lugar. Asimismo, en el Lobtag corpus, se encontraron resultados parecidos, ya que, de los 266 ejemplos de genitivo agente con núcleo subordinado inanimado, el 64,2 \% de ellos pueden relacionarse con la actividad humana y en el 35,8 \% de los casos este vínculo no se produce. Si se ven algunos ejemplos de ambos corpus, puede notarse que el núcleo subordinado es un nombre inanimado el cual, en cierto modo, puede relacionarse con la actividad humana.

(30) Brown corpus

The Administration's proposals

The board's action

The Church's stance

The Spanish Armada's threats

The Pentagon's announcement

The Navy's efforts

The state's condemnation of a man

A company's practices and opinions

(31) Lobtag corpus

The nation's greatest need

The government's frequent appeals to the electorate

A.E.U. national committee's demand

The federation's racial policies

The town's fight for its rights

The Church's principles

The school's job in school hours

The new party's evolution towards an Anti-Semitic fascism 
El genitivo agente como la interpretación semántica más prototípica en inglés: un estudio de corpus

Este subgrupo pone de manifiesto que la construcción de genitivo suele aparecer con relativa frecuencia con nombres inanimados como núcleos subordinados, pero en la gran mayoría de los casos que esto ocurre, este nombre inanimado puede ser vinculado, en cierto modo, con la actividad humana, puesto que una compañía o un gobierno, por ejemplo, la constituyen personas. En definitiva, se puede concluir que, atendiendo a los datos obtenidos del estudio de corpus realizado, la construcción de genitivo tiende a aparecer con nombres animados y humanos, aunque esto no impide, como se ha comprobado, que se dé con nombres no animados y no humanos.

La última subclasificación realizada atendiendo a los rasgos semánticos del núcleo subordinado de los ejemplos de genitivo agente engloba a los nombres de animales $\mathrm{y}$, por tanto, el núcleo presenta los rasgos [+ animado] y [- humano]. Este subgrupo es muy poco frecuente en ambos corpus, pues representa solo el 0,3\% de los resultados de agente en el Brown corpus y el 0,6 \% en el Lobtag corpus, como se aprecia en (32):

(32) The lioness' point of view

A fox's life

A tired horse's plodding step

A snail's crawl

The lion's roar

The bird's disappearance

Animals' reactions to sounds

The grey squirrels' sins

Ante estos ejemplos, se debe aclarar que muchos de estos ejemplos fueron clasificados como casos de genitivo agente, porque han sido interpretados en sentido figurado. Si se analiza más detenidamente alguno de ellos, al tomar como muestras the lioness' point of view y the grey squirrels' sins, se aprecia que la acción de «pecar» es un acto más relacionado con el hombre que con los animales y que el «punto de vista» es algo que se vincula más a los humanos que a los animales, pues los primeros son, a priori, más aptos para expresar una opinión que los últimos, aunque esto no impide que un animal pueda poner en claro mediante su comportamiento cuál es su «punto de vista» u «opinión». Por esta razón, dichos resultados fueron interpretados como agentes, aunque se es consciente de que hay que entenderlos en sentido figurado para poder ser comprendidos de tal modo.

\subsection{El genitivo agente y la nominalización como procesos de «empaquetado» de información}

Se debe aclarar que, lo que a continuación se expone en relación con la condensación de la información, no solo es aplicable al genitivo agente, sino que es propio de la estructura de genitivo en general, sin que la interpretación semántica varíe significativamente lo dicho en las páginas siguientes. No obstante, es relevante establecer esta relación con el genitivo agente, pues esta es, precisamente, la relación semántica más frecuente en ambos corpus, además de ser, como se verá a continuación, la que mejor pone de manifiesto este proceso.

Como se ha demostrado en el apartado anterior, el análisis de ambos corpus revela que la interpretación semántica más frecuente y prototípica del genitivo inglés es el genitivo agente y no el de posesión. Esto lleva a preguntarse cuál puede ser la razón de este hecho. Desde la mirada expuesta en este estudio, la construcción 
El genitivo agente como la interpretación semántica más prototípica en inglés: un estudio de corpus de genitivo en inglés solo tiene una función semántica en la actualidad, que es la de relacionar dos referentes o entidades, es decir, el sintagma subordinado en genitivo (NP's) y el núcleo superordinado del que depende (Stefanowitsch, 2003). Ahora bien, esta relación marcada por el genitivo puede interpretarse de diversas maneras atendiendo no solo al contexto, sino también a factores, principalmente, léxicos y pragmáticos (Moldovan et al., 2004; Vikner \& Jensen, 2002). El hecho de que sea el genitivo agente la más frecuente de estas interpretaciones y su cierta analogía con la interpretación de roles en una oración lleva a plantear que quizás uno de los cometidos de la construcción de genitivo actual sea, precisamente, el de informar como lo hace una oración; en otras palabras, estableciendo quién hace una determinada acción y qué es lo que se hace, siendo esta la estructura más simple de una oración (sujeto + verbo). Lo que sucede es que el genitivo haría esto de una manera más abreviada y sobre todo distinta, de tal modo que desde el punto de vista comunicativo sería lo mismo decir John's criticism of the book que John criticized the book. Tal vez, el hecho de que aparezca el genitivo hace que la relación entre los dos referentes, (NP's) y (N), sea más estrecha que la que se establece entre el sujeto y el verbo de una oración y esto es lo que, precisamente, quiere comunicar el hablante cuando elige la estructura de genitivo y no la de oración.

El fenómeno lingüístico denominado nominalización, como se ha visto anteriormente, consiste en convertir en nombres otras palabras que normalmente no son nombres, es decir, consiste en transformar en nombres, principalmente, verbos (to get sick $\rightarrow$ illness) y conjunciones o conectores (because $\rightarrow$ reason) (Eggins, 1994). Este proceso, según Eggins (1994), es propio de la lengua escrita y por medio de él, se pueden organizar los textos en términos de ideas, razones, causas, etc. Asimismo, la nominalización permite al escritor eludir la dinámica de las secuencias propias de la lengua hablada, donde el uso de verbos para denotar una acción se hace casi inevitable.

Este fenómeno está muy relacionado con el mecanismo de «empaquetado» de la información tan característico de la lengua escrita, donde el escritor tiene la posibilidad ilimitada de reorganizar y corregir las ideas a su voluntad. Smit (2007, pp. 91-92) determina que el término de «empaquetado de la información» fue acuñado por Chafe en 1976, quien explica que «cuando se comunica una proposición, el hablante puede realizarla por medio de diferentes estructuras según sus evidencias sobre lo conocido por el oyente». Según esta definición, se puede inferir que el empaquetado de la información es la elección estructural que el hablante hace en un determinado momento para comunicar un mensaje al oyente (Vallduví, 1993).

El proceso de nominalización, por tanto, permite condensar el contenido léxico de una oración, lo que está estrechamente vinculado con el potencial que tiene el sintagma nominal en inglés de hacer lo mismo. En ese sentido, se insiste en que se analizaron dos corpus de lengua escrita, lo que hace suponer que los diversos autores han utilizado la construcción de genitivo como un proceso más de «empaquetado» de la información, como es la propia nominalización. Asimismo, cabe destacar el hecho de que la misma ofrece numerosas ventajas no solo sintácticas, sino semánticas y pragmáticas (Mackenzie, 1996). Desde el punto de vista sintáctico, la nominalización conlleva una reducción en la complejidad sintáctica. De entre las ventajas pragmáticas, el autor resaltó que la «nominalización favorece la condensación de la información» (Mackenzie, 1996, p. 7), y señaló que un sintagma nominal con nominalización se caracteriza semánticamente por presentar una connotación más abstracta que 
El genitivo agente como la interpretación semántica más prototípica en inglés: un estudio de corpus cuando se utiliza la forma verbal. Según este autor, el emplear la nominalización de un verbo consigue presentar los hechos de manera más imprecisa que formulando una oración con la forma verbal, porque al usar el sintagma nominal se puede conseguir dejar fuera la información relativa a la persona o entidad que realiza la acción del verbo.

Con respecto a la construcción de genitivo, este compendio de la información se presenta de la siguiente manera: en una oración siempre se encuentran unos participantes y una acción, es decir, unos argumentos y un predicado. Según lo expuesto anteriormente, se puede decir que un sintagma nominal en genitivo (NP's) codifica un estado de cosas (state of affairs) de modo similar, aunque no exactamente idéntico, a como lo hace una oración. Al observar el ejemplo (33), se presenta una oración con dos argumentos o participantes y una acción o predicado.

(33) Russia detonated a super bomb.

$\frac{\text { Russia }}{\text { Argumento }} \quad \frac{\text { detonated }}{\text { Predicado }} \quad \frac{\text { a super bomb }}{\text { Argumento }}$

En un sintagma nominal con genitivo, se pueden encontrar los mismos argumentos; de hecho, el propio sintagma en genitivo (NP's) identifica uno de ellos, mientras que el otro puede aparecer realizado por medio de una complementación con of (of + NP) como lo demuestra el siguiente ejemplo del Brown corpus.

(34) Russia's detonation of a super bomb.

$\frac{\text { Russia's }}{\text { Argumento }} \quad \frac{\text { detonation }}{\text { Predicado }} \quad \frac{\text { of a super bomb }}{\text { Argumento }}$

Taylor (1996), por su parte, distinguió dos paradigmas bastantes extensos con respecto a las nominalizaciones en inglés. El primero de ellos lo ejemplifica con el sustantivo destruction:

(35) a. The enemy's destruction of the city

b. The city's destruction by the enemy

c. The city's destruction (genitivo afectado)

Según estos ejemplos, se pueden resaltar varios aspectos:

(A) El sintagma en genitivo (NP's) puede interpretarse como agente (35a) o como afectado (35b, 35c).

(B) La interpretación de agente solo puede hacerse cuando aparece un segundo argumento, realizado en este caso por un complemento con of (35a).

(C) Si se elimina este segundo argumento o complemento con of, entonces solo se puede interpretar como genitivo afectado (35c).

Al igual que destruction, otros muchos nombres (invasion, assassination, dismissal, etc.) presentan estas propiedades, es decir, tienen en común que se derivan de un verbo que denota situaciones en las cuales una entidad con el rol de agente realiza alguna acción que afecta o recibe otra entidad la cual tiene el rol de afectado. Estos nombres son denominados por Taylor como «nombres de acción» (action nouns) (1996), de los cuales el corpus muestra numerosos ejemplos. Como puede observarse, en cada uno de ellos aparece un segundo argumento introducido realizado por un complemento con la preposición of. 
El genitivo agente como la interpretación semántica más prototípica en inglés: un estudio de corpus

(36) Brown corpus

Robert McEnroe's adaptation of Maurice Walsh's film

Oersted's first demonstration of his discovery

Israel's 1956 invasion of Egypt

The state's condemnation of a man

Katherine Squire's vigorous characterization of a farm mother

Fromm's citation of the other diagnosticians

(37) Lobtag corpus

President Kennedy's rejection of it

West Germany's economic domination of Western Europe

Jocelyn Brooke's investigation of Proust and Joyce

Roosevelt's assumption of office

Matthew's interpretation of the material

The company's valuation of each employee

El segundo paradigma que Taylor (1996) propuso estaría integrado por los nombres que se derivan de los llamados «verbos cognitivos» (cognitive verbs). Estos verbos denotan una situación en la cual una entidad, presumiblemente un ser humano, presenta un estado cognitivo o emocional con respecto a otra entidad, típicamente el objeto directo del verbo. Ejemplos de este tipo de verbos son: (to) admire, (to) distrust, (to) fear, (to) love, (to) know, etc. En el corpus, también se han hallado casos que pueden ilustrar este paradigma.

\section{(38) Brown corpus}

His mother's love for him

Morse's knowledge of what Mrs. Borden told Bridget

Bultmann's own definition of myth

Ann's own description of the scene

The suspended diplomat's fears to rest

Hegel's profound admiration for the insights of the Greek tragedians

(39) Lobtag corpus

Morse's knowledge of what Mrs. Borden told Bridget.

Khlebnikob's love of horrors

Lautrec's vision of his women

Anabella's detestation of Mr. Hobhouse

Mrs. Harris's opinion of me

Coleridge's definition of the secondary imagination

Estos paradigmas sugieren, pues, la posibilidad de que la nominalización de un verbo pueda servir como un mecanismo o recurso que se emplea en la lengua con el fin de condensar la información que se está dando. De este modo, todos los casos anteriormente presentados ofrecen la misma información que si se estuviera ante una oración. Compárense los siguientes ejemplos:

(40) a. Anabella's detestation of Mr. Hobhouse

b. Anabella detests Mr. Hobhouse.

(41) a. President Kennedy's rejection of it

b. President Kennedy rejected it.

En los ejemplos (40a) y (41a) se presenta la información mediante un sintagma nominal en el que una de las entidades aparece marcada por el genitivo (NP's), mientras que en los ejemplos (40b) y (41b) la misma 
El genitivo agente como la interpretación semántica más prototípica en inglés: un estudio de corpus información se descubre por medio de una oración.

En definitiva, y a modo de conclusión, se puede decir, que una de las peculiaridades de la estructura de genitivo es la de poder condensar la información y presentar esta de un modo más conciso y escueto, pero sin dejar de significar y comunicar, esencialmente, lo mismo que una oración.

\section{Conclusión}

En este artículo se ha puesto de manifiesto que el genitivo agente, a pesar de lo que tradicionalmente se ha mantenido, es la interpretación semántica más prototípica y frecuente de la construcción del genitivo. Asimismo, se pudo comprobar que esto sucede en las dos variedades del inglés tenidas en cuenta en el análisis de corpus, es decir, tanto en el inglés americano como en el inglés británico.

Los resultados obtenidos del estudio del corpus revelan que son más frecuentes los ejemplos de genitivo agente en los que aparece como núcleo superordinado un nombre de acción o un nombre derivado de un verbo, que aquellos en los que la acción se sobreentiende, pero no aparece de manera explícita. Igualmente, con respecto a los rasgos semánticos del núcleo subordinado, se constató que el genitivo agente es más reiterado cuando el núcleo es un nombre con el rasgo léxico [+ humano] y mucho más frecuente cuando dicho núcleo es un nombre propio de persona. Indudablemente, un futuro estudio más detallado sobre los nombres que presentan el rasgo [humano] y [- animado] aportaría resultados muy interesantes desde el punto de vista lingüístico.

En el último apartado, se expuso la vinculación que tanto el genitivo, en general, como el genitivo agente, en particular, tiene con el mecanismo de empaquetado de la información, de la que se concluyó que, mediante la construcción del genitivo se puede compendiar la información en el discurso, de tal manera que se podría comunicar, básicamente, lo mismo que en una oración, solo que esto se haría de un modo estructuralmente diferente. 
El genitivo agente como la interpretación semántica más prototípica en inglés: un estudio de corpus

\section{Referencias bibliográficas}

1. Alexiadou, A., \& Schäfer, F. (2006). Instrument Subjects Are Agents or Causers. In D. Baumer, D. Montero \& M. Scanlon (Eds.), Proceedings of WCCFL 25 (40-48). Somerville, MA: Cascadilla Proceedings Project.

2. Anthony, L. (2013). A Critical Look at Software Tools in Corpus Linguistics. Linguistic Research, 30(2), 141161.

3. Barker, C. (1995). Possessive Descriptions. Stanford: CSLI Publications.

4. Chomsky, N. (1970). Remarks on Nominalization. In A. Roderick \& P. Rosenbaum (Eds.), Readings in English Transformational Grammar (pp. 184-221). Waltham MA: Ginn.

5. Christophersen, P. \& Sandved, A. O. (1969). An Advanced English Grammar. London: MacMillan.

6. Crystal, D. (1997). A Dictionary of Linguistic and Phonetics. Oxford: Blackwell.

7. Curme, G. O. (1935). Parts of Speech and Accidence. Boston: D. C. Heath and Company.

8. Curme, G. O. (1947). English Grammar. The Principles and Practice of English Grammar Applied to PresentDay Usage. New York: Barnes \& Noble, INC.

9. Eggins, S. (1994). An introduction to systemic functional linguistics, London: Pinter Publishers.

10. Hawkins, R. (1981). Towards an Account of the Possessive Constructions: NP's N and the N of NP. Journal of Linguistics, 17, 247-269.

11. Hogg, R. M. (1992). The Cambridge History of the English Language. Cambridge: Cambridge UP.

12. Huddleston, R. \& Pullum, G. K. (2002). The Cambridge Grammar of the English Language. Cambridge: Cambridge UP.

13. Johansson, S., Leech, G. N., \& Goodluck, H. (1978). The Lancaster-Oslo/Bergen Corpus of British English (LOB Corpus). Department of English: Oslo UP.

14. Kučera, H. \& Francis, W. N. (1967). The Brown University Standard Corpus of Present-Day American English (Brown Corpus). Providence: Brown University.

15. Langacker, R. W. (1991). Foundations of Cognitive Grammar. Stanford, California: Stanford UP.

16. Lyons, C. (1986). The Syntax of English Genitive Constructions. Journal of Linguistics, 22, 123-43.

17. Mackenzie, J. L. (1996). English Nominalizations in the Layered Model of the Sentence. Complex Structures: A Functionalist Perspective, 325-353.

18. McCawley, J. D. (1998). The Syntactic Phenomena of English. Chicago: U of Chicago Press.

19. Moldovan, D., Badulescu, A., Tatu, M., Antohe, D., \& Girju, R. (2004). Models for the Semantic Classification of Noun Phrases. In Proceedings of the Computational Lexical Semantics Workshop at HLT-NAACL 2004 (pp. 60-67).

20. Nesfield, J. C. (1912). Modern English Grammar. London: MacMillan.

21. Quirk, R., Greenbaum, S., Leech, G., \& Svartvik, J. (1985). A Comprehensive Grammar of the English Language. London: Longman. 
El genitivo agente como la interpretación semántica más prototípica en inglés: un estudio de corpus 22. Sledd, J. H. (1959). A Short Introduction to English Grammar. Glenview, Illinois: Scott, Foresman and Co. 23. Smit, N. (2007). Information Packaging in Functional Discourse Grammar. ALFA: Revista de Linguística, 51(2), 91-118.

24. Stefanowitsch, A. (2003). Constructional Semantics as a Limit to Grammatical Alternation: The Two Genitives of English. Topics in English Linguistics, 43, 413-444.

25. Taylor, J. R. (1994). «Subjective» and «Objective» Readings of Possessor Nominals. Cognitive Linguistics, 5, 201-242.

26. Taylor, J. R. (1996). Possessives in English. An Exploration in Cognitive Grammar. New York: Clarendon Press.

27. Vallduví, E. (1993). Information Packaging: A Survey. HCRC Publications, University of Edinburgh.

28. Vikner, C., \& Jensen, P. A. (2002). A Semantic Analysis of the English Genitive. Interaction of Lexical and Formal Semantics. Studia Linguistica, 56(2), 191-226. 